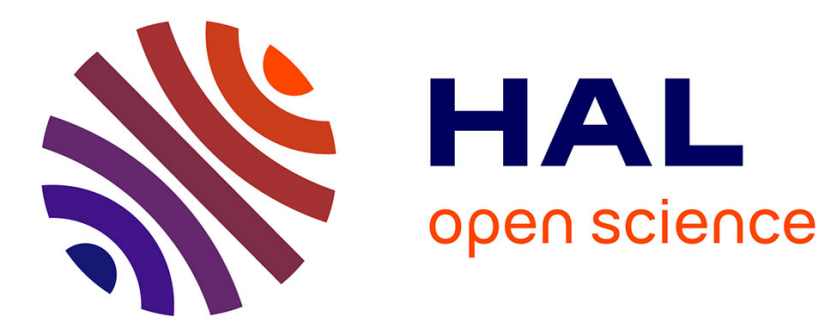

\title{
Robust error on magnetotelluric impedance estimates
}

Pierre Wawrzyniak, Pascal Sailhac, Guy Marquis

\section{To cite this version:}

Pierre Wawrzyniak, Pascal Sailhac, Guy Marquis. Robust error on magnetotelluric impedance estimates. Geophysical Prospecting, 2013, 61, pp.533-546. 10.1111/j.1365-2478.2012.01094.x . hal00930099

\section{HAL Id: hal-00930099 \\ https://hal.science/hal-00930099}

Submitted on 28 Jan 2014

HAL is a multi-disciplinary open access archive for the deposit and dissemination of scientific research documents, whether they are published or not. The documents may come from teaching and research institutions in France or abroad, or from public or private research centers.
L'archive ouverte pluridisciplinaire $\mathbf{H A L}$, est destinée au dépôt et à la diffusion de documents scientifiques de niveau recherche, publiés ou non, émanant des établissements d'enseignement et de recherche français ou étrangers, des laboratoires publics ou privés. 


\title{
Robust Error on magnetotelluric impedance estimates
}

\author{
Pierre Wawrzyniak ${ }^{1 *}$, Pascal Sailhac ${ }^{1}$ and Guy Marquis ${ }^{1,2}$ \\ ${ }^{1}$ Ecole et Observatoire des Sciences de la Terre, Strasbourg, France, and ${ }^{2}$ Shell International Exploration and Production, Houston, USA
}

Received November 2010, revision accepted December 2011

\begin{abstract}
We propose here a new, robust, methodology to estimate the errors on a magnetotelluric (MT) impedance tensor. This method is developed with the bounded influence remote-reference processing (BIRRP) code in a single site configuration. The error is estimated by reinjecting an electric field residual obtained after the calculation of an impedance tensor into a tensor function calculation procedure. We show using synthetic examples that the error tensor calculated with our method yields a more reliable error estimate than the one calculated from Jackknife statistics. The modulus of realistic error estimates can be used as a quality control and an accurate inversion constraint of MT surveys. Moreover, reliable error estimates are necessary for new applications of MT to dynamic subsurface processes such as reservoir monitoring.
\end{abstract}

Key words: Electromagnetics, Passive methods, Monitoring, Data processing, Signal processing.

\section{INTRODUCTION}

Magnetotellurics (MT) uses natural electromagnetic field variations recorded at the Earth's surface to image the electrical resistivity of the ground subsurface. This is a prospecting method for which sources are ionospheric currents due to solar wind-magnetosphere interactions at frequencies under 1 $\mathrm{Hz}$ and lightning activity propagating through the atmosphere at higher frequencies.

One can show (e.g., Vozoff 1987) that the horizontal electric and magnetic fields are related through the socalled impedance tensor $Z$. This tensor is estimated from the recorded time-series as transfer functions between the magnetic $H$ and electric $E$ field horizontal components (Sims 1971). The impedance $Z$ is also a function of the subsurface resistivity.

The estimation of $Z$ is arguably the most significant stage of MT data processing. Most practitioners follow some form of least-squares fitting procedure to subsets of data segments

*E-mail: pierre.wawrzyniak@unistra.fr in the Fourier domain (e.g., Vozoff 1987). This approach to estimate the MT impedance tensor relies on several assumptions:

- The incident wave is a plane wave, i.e., MT geomagnetic sources are located far from the site of MT measurement.

- The signal is assumed to be stationary during the estimation period.

- The subsurface resistivity is also assumed to be stationary during the estimation period.

- The noise on the EM fields is Gaussian.

Obviously, these conditions are not always strictly met: non-stationary phenomena exist on the magnetic field $H$ and long-term subsurface changes may induce a time variability of $Z$. Moreover, the data are often affected by cultural noise induced by power lines, moving vehicles or industrial infrastructure. In most estimation procedures, any departure from these ideal conditions will be considered as noise and modelled as Gaussian residuals of the electric field.

In order to address some of these noise problems, Gamble, Goubau and Clarke (1979) introduced the remote reference technique where a second MT site is used to remove the influence of noise included in the local MT site magnetic field that may bias impedance estimates. Impedance tensor estimation 
remained however based on band-averaged and time-averaged cross-correlations in the Fourier domain (Sims, Bostick and Smith 1971).

Adaptative robust tensor estimation procedures were developed in the 1980 's, by downweighing high residuals and singular data (Larsen 1980; Egbert and Booker 1986; Chave, Thomson and Ender 1987). By comparing these different approaches, Jones et al. (1989) illustrated the improvement brought by the use of robust data processing methods.

On a more general level, a measurement is valid and can be used for law fitting when one also gets a reliable measure of its accuracy. Also error estimates on MT impedances are often used as weighting factors in MT inversions. In standard robust MT methods, errors are defined by the variance on the population of $Z$ estimates. Chave and Jones (1997) showed that conventional distribution-based error estimates are often biased. Because they were considered to be less sensitive to non-stationarity and depart from Gaussian error distributions, Chave and Thomson (1989) replaced standard variance by Jackknife variance (introduced by Efron 1982 and Efron and Gong 1983). Improved robust methods should allow the MT user to obtain more stable and more reliable MT impedance estimates.

The question of the stability of MT estimates and their associated errors has been discussed by Eisel and Egbert (2001). They showed that Jackknife variance errors did not reliably estimate the variability of the successive estimates over the full range of MT frequencies. They suggest that day-to-day variability is the best way to estimate impedance accuracy. More recently, Hanekop and Simpson (2006) suggested that deviations from the plane wave assumption used in the MT method leads to errors on impedance estimates that will not be reflected in the standard error bars.

In this article, we introduce in detail a new type of error estimate, the error tensor. It is based on a robust leastsquares regression between the horizontal magnetic field and the residuals of the electric field obtained from the impedance estimation.

For testing the reliability of the proposed error calculation, we first consider a synthetic data set, where a real timeseries of the magnetic field $h$ is convolved with two synthetic impedance models: a homogeneous half-space and a 1D layered resistivity. A real time-series of the magnetic field is used instead of a synthetic superposition of pure or singular oscillations because our aim is to comfort the robustness of the method with realistic non-stationary behaviours. We compare the true and estimated quantities to assess the accuracy of the estimation method. We focus on the modulus of the error tensor since the apparent resistivity is defined through the modulus of the impedance tensor. Then, several levels of Gaussian noise are added on electric field time series and the error estimator is tested again.

Second, we consider a real data set on a site where the resistivity does not change in time, so that any variation of the impedance should be interpreted as a limit of the processing method. We study the variability of successive estimates over the day and compare it with error estimates. Reliable error estimates ought to provide the upper and lower bounds of the $Z$ variability.

\section{METHODOLOGY}

\section{Estimating the impedance tensor}

In the time domain, $e_{i}(t)$ and $h_{j}(t)$ are related by a convolution equation involving the impedance tensor $z_{i j}(t)$ :

$e_{i}(t)=\sum_{j} z_{i j}(t) * h_{j}(t)+\mu_{i}(t)$,

where $i, j$ are direction indices (say, $1=x, 2=y$ ) and the asterisk * denotes the convolution product (in the time domain). While $e_{i}(t)$ and $h_{j}(t)$ can be considered as random but observable variables, $z_{i j}(t)$ is non-random and unobservable and $\mu_{i}(t)$ is the noise.

In most processing techniques (e.g., Chave et al. 1987; Egbert 1997), the electric and magnetic fields are transformed into the Fourier domain (respectively $E_{i}(f)$ and $\left.H_{j}(f)\right)$ and convolution equation (1) becomes, in discrete form:

$E_{i}\left(f_{k}\right)=\sum_{j} Z_{i j}\left(f_{k}\right) H_{j}\left(f_{k}\right)+N_{E_{i}}\left(f_{k}\right)$,

where $N_{E_{i}}\left(f_{k}\right)$ is the electric field misfit and is assumed to follow normal statistics with zero mean.

The approach introduced here can be used with any transfer function estimation technique. Here we consider the bounded influence remote-reference processing (BIRRP) code (Chave and Thomson 2004), which solves equation (2) using weighted least squares (LSQ) within a series of time windows (called 'delete-ones'). BIRRP is used here in single site (SS) configuration. To improve the accuracy, the length of these time windows is chosen to be inversely proportional to the target frequency $f_{k}$ (meaning time-frequency resolution comparable to that of the wavelet transform (Zhang et al. 1997)). Thus solving equation (2) within all subsets yields a unique estimate $Z_{i j}\left(f_{k}\right)$ and a series of residuals on the electric field $\Delta E_{i}\left(f_{k}\right)$. This population of residuals is considered and compared to a reference statistical model in order to detect outliers, apply 
Jackknife filtering and define weighted factor for the LSQ. One finally obtains a robust estimate for $Z_{i j}\left(f_{k}\right)$.

\section{The error tensor}

In addition to a robust estimate for $Z_{i j}\left(f_{k}\right)$, BIRRP also provides the user with the spreading parameter of the jackknife estimates population (related to the variance). This is classically used as an estimate of the uncertainty on $Z_{i j}\left(f_{k}\right)$ but it is biased in cases for which low geomagnetic activity or anthropic noise involves a population of $Z_{i j}\left(f_{k}\right)$ estimates with a small variance but with a mean far from the true value. These errors are mostly underestimated as they are physically related to the variability of jackknife estimates.

To address this problem, we propose here the use of a new estimator for errors on the MT impedance tensor: the error tensor, defined on the basis of residuals of the electric field.

Let us call $Z_{i j}^{\text {est }}\left(f_{k}\right)$ the estimated impedance tensor at a frequency $f_{k}$. By product with the magnetic field this yields to:

$E_{i}^{P}\left(f_{k}\right)=\sum_{j} Z_{i j}^{\text {est }}\left(f_{k}\right) H_{j}\left(f_{k}\right)$

where $E_{i}^{P}\left(f_{k}\right)$ is the predicted electric field in the Fourier domain.

By subtracting equation (3) from equation (2), we obtain the following relation for the residuals:

$\Delta E_{i}\left(f_{k}\right)=\sum_{j} \Delta Z_{i j}\left(f_{k}\right) H_{j}\left(f_{k}\right)+N_{E_{i}}\left(f_{k}\right)$.

Here, $\Delta E_{i}\left(f_{k}\right)$ is the difference between the observed and produced electric fields:

$\Delta E_{i}\left(f_{k}\right)=E_{i}\left(f_{k}\right)-E_{i}^{P}\left(f_{k}\right)$,

and the error tensor $\Delta Z_{i j}\left(f_{k}\right)$ is a $2 \times 2$ tensor defined as the difference between the true impedance $Z_{i j}$ and the estimated tensor:

$\Delta Z_{i j}\left(f_{k}\right)=Z_{i j}^{e s t}\left(f_{k}\right)-Z_{i j}\left(f_{k}\right)$.

The assumption of a normal distribution of $\Delta E_{i}$ is first used to determine the impedance components $Z_{i j}$ by solving equation (2) and is moved to similar linear equation (4) to be solved to determine impedance errors $\Delta Z_{i j}$. The estimation of the error tensor can be made with classical robust MT impedance computation techniques by passing $\Delta E_{i}\left(f_{k}\right)$ back to the time domain and then using the time-series $\left(\Delta e_{i}(t), h_{j}(t)\right)$ as input to the transfer function estimation code. The error tensor is computed in the same way as the MT tensor itself and its components can be considered as error estimates on $Z_{i j}^{e s t}\left(f_{k}\right)$.

The error tensor is defined by the residuals from the regression that yields to $Z_{i j}^{\text {st }}\left(f_{k}\right)$. For the presentation that follows, we introduce three error notations:

1. $\Delta Z_{i j}^{\text {sst }}$ denotes the new robust error estimates (solution to equation (4))

2. $\Delta Z_{i j}^{\text {birp }}$ is BIRRP's own error estimates based on the socalled Jackknife variance of impedance estimates

3. $\Delta Z_{i j}^{\text {obs }}$ is the true error observed in our synthetic tests (by direct use of the definition in equation (6) with the true impedance tensor)

\section{Parameters of sliding windows}

We consider successive runs of BIRPP, in a single station (SS) processing configuration, on data acquired within a single day to provide several estimates, each being relative to a record duration $D$. This duration is related to the period $T_{0}$ (in seconds as for $D$ ), to the number of delete-ones (elemental sliding windows for each estimate) $N$ and to the number of periods within each delete-one $\mathrm{K}$ :

$D=c N K T_{0}$,

where $c$ is an overlapping factor (dimensionless and strictly lower than unity).

For simplicity in this paper, we focus on 3 values of $T_{0}$ : $5 \mathrm{~s}, 0.5 \mathrm{~s}$ and $0.05 \mathrm{~s}$. These periods are used for all synthetic and real data cases; they are related to the skin depth $\delta \approx 503 \sqrt{T_{0} \rho_{a}}$ (where $\delta$ is in metres, $T_{0}$ is in seconds and the apparent resistivity $\rho_{a}$ is $\Omega$.m) so they address a significant range of depth samplings. They are considered for the estimates of both the MT and error tensors $\left(Z_{i j}^{\text {est }}\left(f_{k}\right)\right.$ and $\Delta Z_{i j}^{\text {est }}\left(f_{k}\right)$ respectively).

For each frequency of interest, the data are decimated to a new sampling rate $f_{s}$. We use the BIRRP code with elemental windows or delete-ones of 3 different lengths (see Table 1), respectively increasing the number $K$ of periods $T_{0}$ per elemental window. The parameter $N$ is not systematically tested: this is the number of elemental windows (delete-ones) for each BIRRP estimate. After a few tests $(N=125,250,500)$, we noted that the most important sensitivity is on parameter $K$ so for all cases considered we will use $N=250$. Tests using different values of $K$ (and corresponding values of $D$ ) are shown for the synthetic homogenous case; for all other applications (synthetic 1D case and the real data case), we use a fixed $K$ of 6 periods per elemental window. 
Table 1 Duration of the windows for $Z$ and $\Delta Z$ estimates in function of the number $K$ of periods To contained in each delete-one (columns), for each period $T_{0}$ of interest (lines) and for a fixed number $N$ of 250 delete-ones

\begin{tabular}{llll}
\hline$T_{0}[\mathrm{~s}]$ & $f_{S}[\mathrm{~Hz}]$ & $K$ & $D[\mathrm{~s}]$ \\
\hline 0.05 & 213.33 & 3 & 38 \\
& & 6 & 77 \\
& & 12 & 154 \\
0.5 & 21.33 & 3 & 384 \\
& & 6 & 768 \\
5 & 2.13 & 12 & 1536 \\
& & 3 & 3840 \\
& & 12 & 7680 \\
& & & 15360 \\
\hline
\end{tabular}

\section{TEST ON SYNTHETIC MODELS}

To test the validity of the error estimation method, we produced a hybrid data set consisting in a real magnetic data (MT source) but a synthetic electric field based on a well-defined impedance model. We chose this approach because:

- if we use a real $(e, h)$ data set, we will not have full knowledge of the true impedance $Z_{i j}$ which is necessary to compute the true errors to be compared to estimated errors;

- if we use a purely synthetic data set, we will be able to know exactly the true impedance $Z_{i j}$ but the time fluctuations and instabilities will not be representative of the complexity and realism of the geomagnetic external variations. Actual non-stationarities, including unknown natural, anthropic noise and deviation from the plane wave assumption of MT sources are required in the synthetic data because they can be a significant cause of errors on impedance estimates. Thus, we use a hybrid data set, i.e. 'semi-synthetic': we use an observed magnetic field $h_{j}(t)$ but we compute a synthetic electric field $e_{i}^{s}(t)$ based upon a simple analytic impedance model in the frequency domain $Z_{i j}^{M}$, which is convolved with the observed magnetic field (equation (3)). The real magnetic data set is a 24-hour time-series acquired at $1024 \mathrm{~Hz}$ on August 5th 2009, during a magnetotelluric survey on the granitic area of Avrillé (Vendée, France). A first set of synthetic data uses a homogeneous resistivity model and a second uses a layered resistivity model. The quality of the impedance tensor and error tensor estimates obtained on one synthetic timeseries is obtained by comparing the statistical behaviour and time variability of the relative errors over the day: true errors $\Delta Z_{i j}^{\text {obs }}\left(f_{k}\right)$, our new error tensor $\Delta Z_{i j}^{\text {est }}\left(f_{k}\right)$ and the errors given by BIRRP $\Delta Z_{i j}^{b i r p}\left(f_{k}\right)$. This approach assumes correlated noise between the electric and magnetic fields, which is supposed to not be the case for the tensor estimation procedure.

\section{The homogeneous case}

First we consider a simple hybrid data set, using an impedance $Z_{i j}^{M}$ corresponding to a homogeneous medium of resistivity $\rho_{M}=750 \Omega . \mathrm{m}$. The wavenumber $k_{M}$ is defined as:

$k_{M}=\sqrt{\frac{\omega \mu_{M}}{2 \rho_{M}}}(1-\mathrm{I})$,

where $\mathrm{I}=\sqrt{-1}, \omega=2 \pi f$ and $\mu_{M}=\mu_{0}$ is the magnetic permeability in the medium classically set to be equal to the free-air permeability (the relative change in the electrical resistivity $\rho_{M}$ is much greater than in $\left.\mu_{M}\right)$. The impedance tensor is a simple antisymmetric tensor with zero diagonal and opposite off-diagonal terms $Z_{i, j}^{M}=(-1)^{i+1} Z_{M}$ where $i, j$ are direction indices and $Z_{M}$ is the medium complex impedance:

$Z_{M}=\frac{\omega \mu_{0}}{k_{M}}=\sqrt{\frac{\rho_{M}}{2 \omega \mu_{0}}}(1+\mathrm{I})$.

\section{Statistical behaviour of true and estimated relative errors}

Let us focus on the results obtained on the $x y$ component (figs 1, 2 and 3); from a statistical point of view, its behaviour is similar to that of the other off-diagonal element $(y x)$, while the diagonal elements are negligible. We consider both histograms of the error estimates normalized to true errors $\left(\left|\Delta Z_{i j}^{\text {birp }}\right| /\left|\Delta Z_{i j}^{\text {obs }}\right|\right.$ and $\left.\left|\Delta Z_{i j}^{\text {est }}\right| /\left|\Delta Z_{i j}^{\text {obs }}\right|\right)$ and relative true errors $\left|\Delta Z_{i j}^{o b s}\right| /\left|Z_{i j}^{o b s}\right|$. We focus on the histogram maximum, providing the most likely value, respectively $X_{0}^{\text {birrp }}, X_{0}^{\text {est }}$ and $X_{0}$ :

- For the true errors $\Delta Z_{x y}^{\text {obs }}, X_{0}$ (see Fig. 1) decreases with increasing $K$ at periods $T_{0}=0.5 \mathrm{~s}$ and $5 \mathrm{~s}$. This dependency on $K$ shows that the longer the delete-ones windows, the better the impedance tensor estimates are. To the contrary, at the shortest period $\left(T_{0}=0.05 \mathrm{~s}\right)$, the longest estimates $(K=12)$ display larger errors than the others. This could be due to the occurrence of short-lived transient noise-sources.

- To analyse their accuracy, one can compare the BIRRP error estimates $\Delta Z_{x y}^{\text {birp }}$ to the true errors $\Delta Z_{x y}^{\text {obs }}$ (see Fig. 2): a large proportion of the Jackknife estimates population $\Delta Z_{x y}^{\text {birp }}$ (red in Fig. 2) underestimates the errors. Errors $\Delta Z^{\text {birp }}$ (or $\Delta Z^{\text {est }}$ ) cannot be considered as reliable when they are lower than the true errors $\Delta Z^{\text {obs }}$. $X_{0}^{\text {birrp }}$ ranges from 0.3 to 3.08 .

- Now comparing the 'error tensor' estimates $\Delta Z_{x y}^{\text {est }}$ to the true errors $\Delta Z_{x y}^{\text {obs }}$ (see Fig. 3), one remarks that most 
Figure 1 Homogeneous case: synthetic data. Histogram of relative true errors $\Delta Z_{x y}^{\text {obs }}$ for 3 periods of estimation $\left(T_{0}=\right.$ $0.05 \mathrm{~s}, 0.5 \mathrm{~s}, 5 \mathrm{~s})$ and several values of $K(K=$ $3,6,12) . X_{0}$ is the most likely value and $n b$ is the number of estimates.

Figure 2 Homogeneous case: synthetic data. Same as Fig. 1 for BIRRP errors normalized by true errors $\left|\Delta Z x y^{\text {birrp }}\right| /$ $\left|\Delta Z x y^{o b s}\right|$. Red curves: part of the histogram with values under unity, indicating an underestimate of errors (associated \% of the population shown); blue curves: values above unity, overestimate.

Figure 3 Homogeneous case: synthetic data. Same as Fig. 2 for our proposed error tensor $\left|\Delta Z x y^{\text {est }}\right| /\left|\Delta Z x y^{o b s}\right|$.
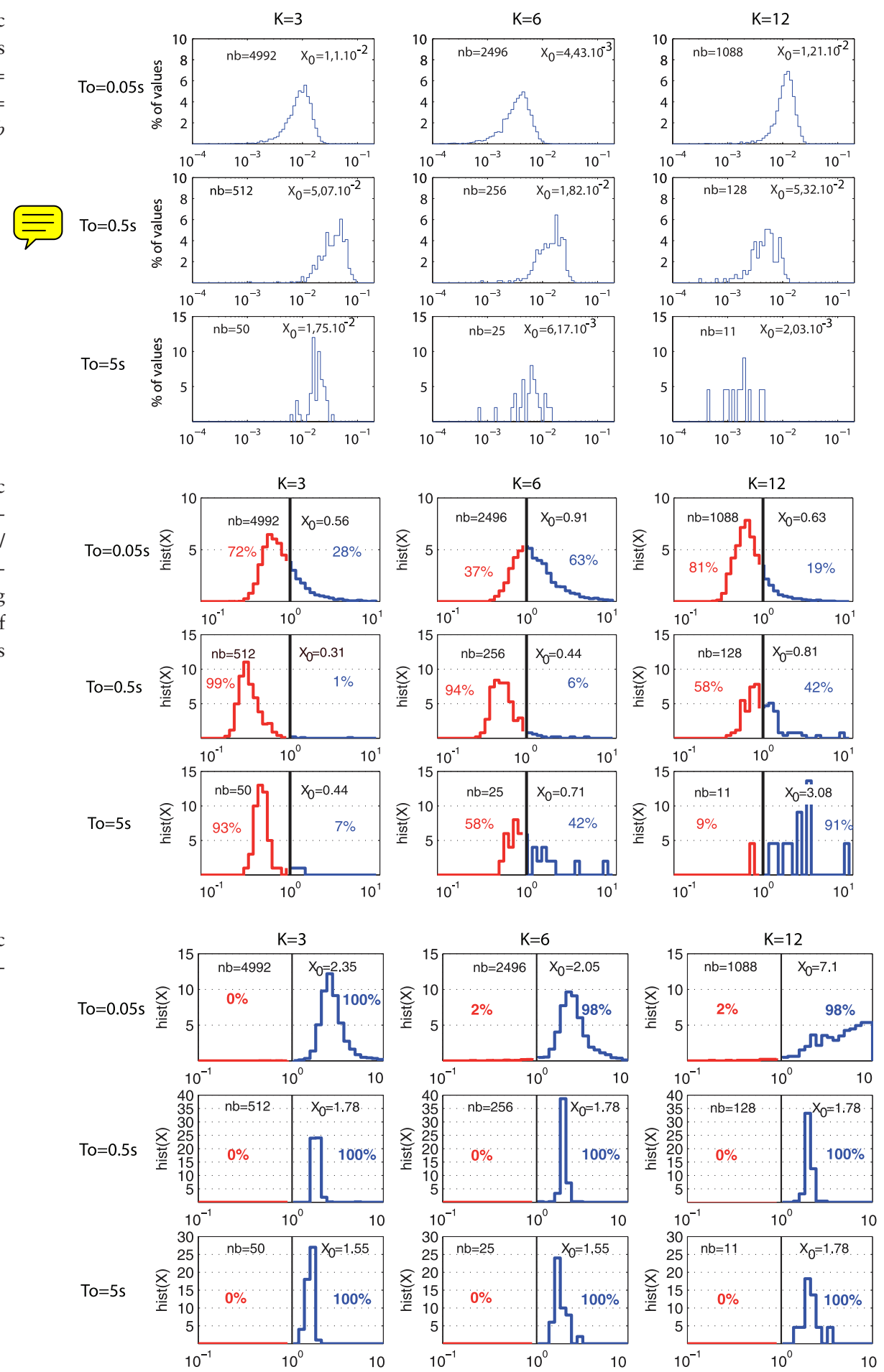

probable values $X_{0}^{\text {est }}$ range from 1.55-2: this is an acceptable overestimation of errors (considering the low relative errors $\Delta Z_{x y}^{\text {obs }}$ shown on figure 1$)$. So $\left|\Delta Z_{x y}^{\text {est }}\right|$ can be used as a diagnostic/quality control (QC) tool during a survey.
One can discuss the 'best' $K$ that leads to both reliable $Z$ and $\Delta Z$ estimates. For the present case, using $K=3$ would be the best choice, because for all periods, all errors $\Delta Z_{x y}^{\text {est }}$ are larger than the true errors so we avoid the pitfall of using 
Table 2 Homogenous case: synthetic data. Correlation coefficients between the true and estimated error time-series for several values of $K$ (number of periods $T_{0}$ contained in each delete-one) at different periods $T_{0}$ of estimation. $C_{i j}^{\text {obs/est }}$ and $C_{i j}^{o b s / b i r r p}$ explained in the text

\begin{tabular}{llllll}
\hline$T_{0}[\mathrm{~s}]$ & $K$ & $C_{x y}^{\text {obs/est }}$ & $C_{y x}^{\text {obs/est }}$ & $C_{x y}^{\text {obs/birrp }}$ & $C_{y x}^{\text {obs/birrp }}$ \\
\hline 0.05 & 3 & 0.83 & 0.61 & 0.41 & 0.46 \\
& 6 & 0.20 & 0.17 & 0.27 & 0.48 \\
& 12 & -0.17 & 0.2 & 0.18 & 0.52 \\
0.5 & 3 & 0.99 & 0.88 & 0.89 & 0.78 \\
& 6 & 0.99 & 0.89 & 0.84 & 0.62 \\
5 & 12 & 0.99 & 0.91 & 0.68 & 0.37 \\
& 3 & 0.98 & 0.97 & 0.7 & 0.66 \\
& 6 & 0.97 & 0.93 & 0.57 & 0.54 \\
& 12 & 0.97 & 0.92 & 0.1 & 0.17 \\
\hline
\end{tabular}

unrealistically small errors in the interpretation. However, this also yields to the largest true errors on the tensor itself (since $\Delta Z_{x y}^{\text {obs }}$ decreases with $K$ ). Thus we would prefer to use $K=6$ for a balance between the error tensor quality (overestimate of errors except $2 \%$ of underestimate for $T_{0}=0.05 \mathrm{~s}$ ) and Tensor quality (lowest true errors). Moreover, we obtain a better time resolution than for the case with $K=12$ : for instance for $T_{0}=$ $0.5 \mathrm{~s}$ ( $2 \mathrm{~Hz}$ frequency), one is able to correctly get one value for each tensor component and its uncertainty every $12 \mathrm{~min}$ $\left(D \approx N K T_{0}\right)$ with $K=6$ or 25 min with $K=12$.

\section{Time variability}

Let us now extend our discussion to both $x y$ and $y x$ components of the impedance tensor. In addition to the histograms of the ratios $\left|\Delta Z_{i j}^{b i r r p}\right| /\left|\Delta Z_{i j}^{\text {obs }}\right|$ or $\left|\Delta Z_{i j}^{\text {st }}\right| /\left|\Delta Z_{i j}^{\text {obs }}\right|$, we also characterize the quality of error estimates by the correspondence between the time fluctuations of estimated errors to the true errors $\left(\Delta Z_{i j}^{\text {birrp }}\right.$ or $\Delta Z_{i j}^{\text {est }}$ to $\left.\Delta Z_{i j}^{\text {obs }}\right)$. We define correlations, for each component $i j$ of the tensor, between observed and new robust errors time series as $C_{i j}^{\text {obs/est }}$ and between observed and classical Jackknife errors time series as $C_{i j}^{o b s / b i r p}$. We use these correlation values $\left(C_{i j}^{o b s / e s t}\right.$ or $\left.C_{i j}^{\text {obs/birrp }}\right)$ to show the accuracy of the estimates in terms of variability (Table 2). Similitude between variability and correlation can be seen by comparing the strong correlation values of $C_{x y}^{\text {obs/est }}$ to the shape of the time fluctuations of the true $\Delta Z_{i j}^{o b s}$ and the estimated errors in Fig. 4.

- Let us consider the values of $C_{i j}^{\text {obs/birrp }}$ (Table 2) and the corresponding plots of error time-series (see Fig. 4). Whatever the value of $K, \Delta Z_{i j}^{b i r p p}$ shows in most cases little correlation with $\Delta Z_{i j}^{\text {obs }}$.
- We note that the accuracy of the variability of the error tensor is evident whatever the parameter settings: at the exception of the shortest periods $\left(T_{0}=0.05 \mathrm{~s}\right.$; for $\left.K=6,12\right)$, $\Delta Z_{i j}^{\text {sst }}$ time-series are strongly correlated with $\Delta Z_{i j}^{\text {obs }}$ (values reach 0.99 in some cases). Practically, for the case where one would like to select the best estimate of $Z$ at a fixed pe$\operatorname{riod} T_{0}$ over a whole day, choosing the estimate associated with the lowest value of $\left|\Delta Z_{i j}^{\text {est }}\right|$ would be reasonable. As the error tensor is based on a regression on the electric field residuals $\Delta e$ of the MT tensor estimation, we suggest that this is probably more representative of the fluctuations of the true magnitude of the error on $\Delta Z_{i j}^{\text {est }}$.

For all $T_{0}$, the best correlations between $\Delta Z_{i j}^{\text {est }}$ and $\Delta Z_{i j}^{\text {obs }}$ are obtained for $K=3$ (see Table 2). This case also corresponds to the worst $Z$ estimates (largest true errors, see Fig. 1).

The plots of both true and estimated errors versus time (Fig. 4) display two regimes associated with daytime and nighttime data. Compared to those from the daytime data, the ( $\left.T_{0}=0.5 \mathrm{~s}, K=6\right)$ estimates based on the night time data are characterized by an increase of errors on the $x y$ element and to a lesser extent on the $y x$ element. Because this case is actually a 'semi-synthetic' data case (real $b$ field + synthetic $Z$ impedance), these patterns are essentially due to internal variability in the properties of $b$ (non-stationarity of the sources, noise and possible deviation from the plane wave hypothesis of MT sources).

\section{The 1D case}

The second family of synthetic data sets is built by using layered (1D) models. The true impedance is computed using classical iterative formulas (e.g., Ward and Hohmann 1987) and accounts for a half-space of resistivity $\rho_{M}=1 \Omega$.m overlain by two layers: the top layer has a fixed resitivity $\rho_{1}=\rho_{M}=$ $1 \Omega . m$ equal to that of the half-space and the intermediate layer resistivity $\rho_{2}$ is allowed to vary and will be used as a sensitivity parameter. Impedance in each layer is defined similarly to that of the homogeneous case (equations (8) and (9)), using the wavenumbers $k_{1}=k_{M}$ and $k_{2}=\sqrt{\frac{\omega \mu_{0}}{2 \rho}}(1-i)$, and scalar impedances $Z_{1}=Z_{M}$ and $Z_{2}=\omega \mu_{0} / k_{2}$.

The first two layers have bottom depths of $h_{1}$ and $h_{2}$ respectively. In order to minimize the frequency dependence with depth, we choose thickness values in relation to the skin depths. To emphasize the effect of the second layer resistivity, we put layer 2 at a depth of one and a half skin depths and gave it a thickness of one skin depth: $h_{1}=1.5 \delta$ and $h_{2}=2.5 \delta$ and we use six different values of layer 2 
Figure 4 Homogeneous case: synthetic data, $K=6$. Relative estimated and true $\Delta Z x y$ (left column) and $\Delta Z y x$ (right column) time-series for 3 periods $T_{0}$ of estimation $\left(T_{0}=0.05 s, 0.5 s, 5 s\right)$ : i) blue $=$ true errors, ii) red $=$ error tensor, iii) green $=$ Birrp error.
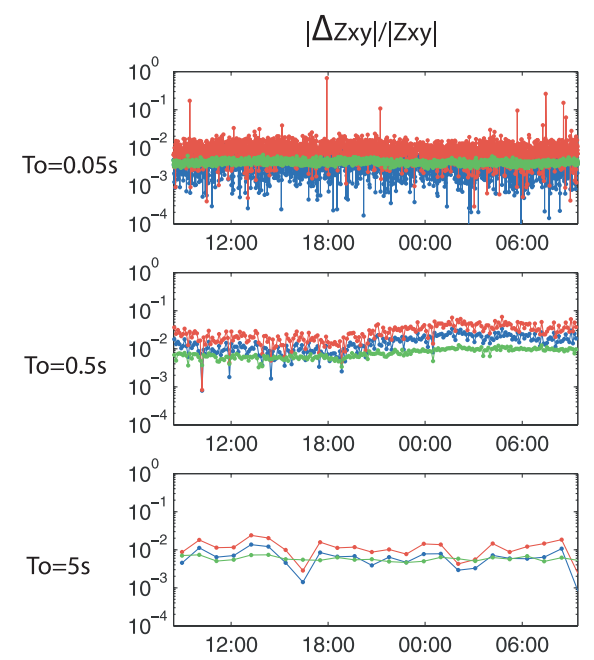

$|\Delta \mathrm{zyx}| /|\mathrm{Zyx}|$
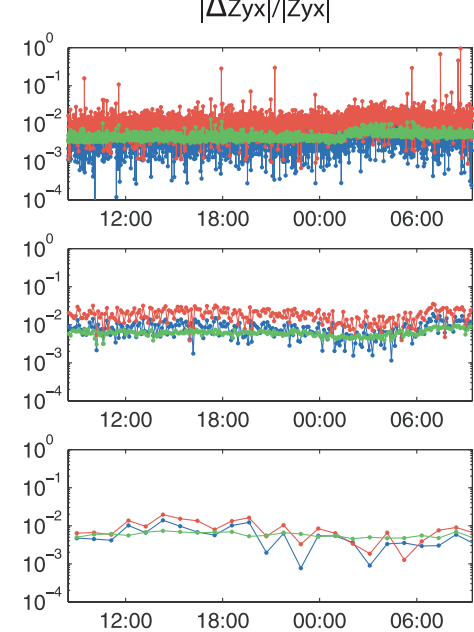

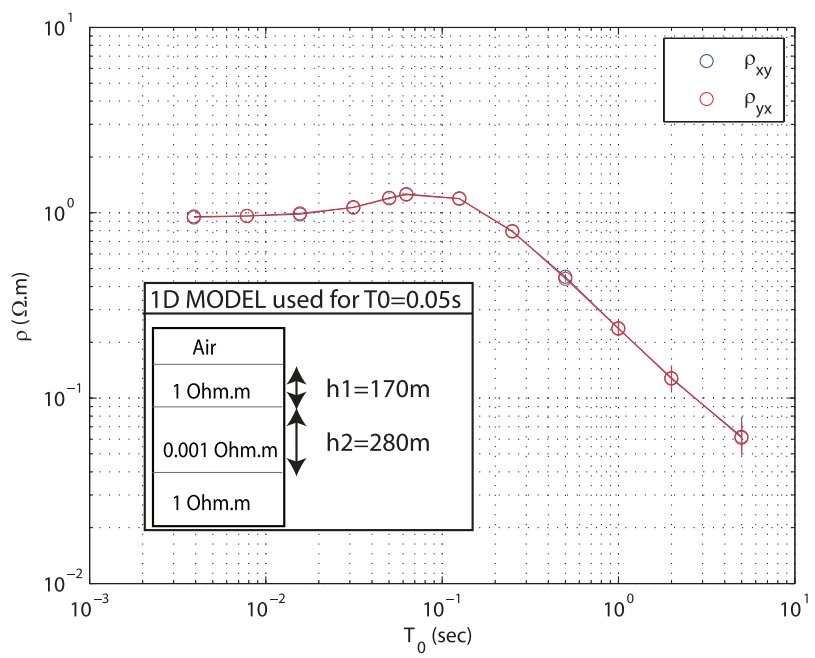

Figure 5 1D case: synthetic data corresponding to one tabular model. Classical MT sounding realized with 3 periods per decade plus 3 additional periods of estimation $\left(T_{0}=0.05 \mathrm{~s}, 0.5 \mathrm{~s}, 5 \mathrm{~s}\right)$. Error bars based on the error tensor are present but very small.

resistivity $\rho_{2}: 10^{-2}, 10^{-1}, 1,10^{1}, 10^{2}$ and $10^{3} \Omega . m$. We fixed the 'binning' parameters $K$ and $N$ to 6 and 250, i.e., the best parameters obtained for the homogeneous case. Among the 18 models created, we show on Fig. 5 a typical MT resistivity sounding curve, computed with the synthetic data and associated with one $1 \mathrm{D}$ model. The quality of the soundings (very low errors) obtained from the other models is similar to this one.

\section{Statistical behaviour of true and estimated relative errors}

As in the homogeneous case, the statistical point of view is discussed using the $x y$ component only.
- Most probable values $X_{0}$ associated with the distribution of true relative errors of $\left|\Delta Z_{x y}^{\text {obs }}\right|$ decrease with increasing period $T_{0}$ (Fig. 6). All errors are small, less than a few percent.

- Values of $X_{0}^{b i r r p}$ show that the population of $\Delta Z_{x y}^{\text {birrp }}$ underestimates $\Delta Z_{x y}^{o b s}$ (see Fig. 7). Looking at histograms of $\left|\Delta Z_{x y}^{b i r p}\right| /\left|\Delta Z_{x y}^{\text {obs }}\right|$, one can observe that for $T_{0}=0.5$ s, $94-96 \%$ of all the associated population is less than unity (i.e., indicating that error estimates are lower than true errors), for $T_{0}=5 \mathrm{~s}$, it is $60-71 \%$ of the population while for $T_{0}=0.05 \mathrm{~s}, 15-40 \%$ of the population is concerned. As in the homogeneous case, a considerable proportion of Jackknife errors underestimate the true errors.

- Histograms of $\left|\Delta Z_{x y}^{e s t}\right| /\left|\Delta Z_{x y}^{\text {obs }}\right|$ and associated values of $X_{0}^{e s t}$ show that, in all cases, error tensor estimates $\left|\Delta Z_{x y}^{\text {est }}\right|$ are larger than the true errors $\left|\Delta Z_{x y}^{o b s}\right|$ (see Fig. 8). For $T_{0}=5 \mathrm{~s}$ and $T_{0}=0.5 \mathrm{~s}, 100 \%$ of all the histograms population is superior to unity. Concerning $T_{0}=0.05 \mathrm{~s}$, $96-98 \%$ of the error population overestimate the true errors. Now, examining in more detail the values of $X_{0}^{\text {est }}$ at $T_{0}=0.5 \mathrm{~s}$ and $T_{0}=5 \mathrm{~s}$, the error tensor overestimates the true errors by factors of 1.75-1.95. For $T_{0}=$ $0.05 \mathrm{~s}$, the overestimates are even higher, ranging from 2.21 to 4 .

Thus, for all frequencies, the error tensor brings a significant improvement of error estimation: true errors are overestimated by a factor 1.7-4 while they were underestimated with Jackknife estimates. However the results for the highest frequency must be used with caution: the overestimate of errors reaches a factor of 4 , a very high value. 


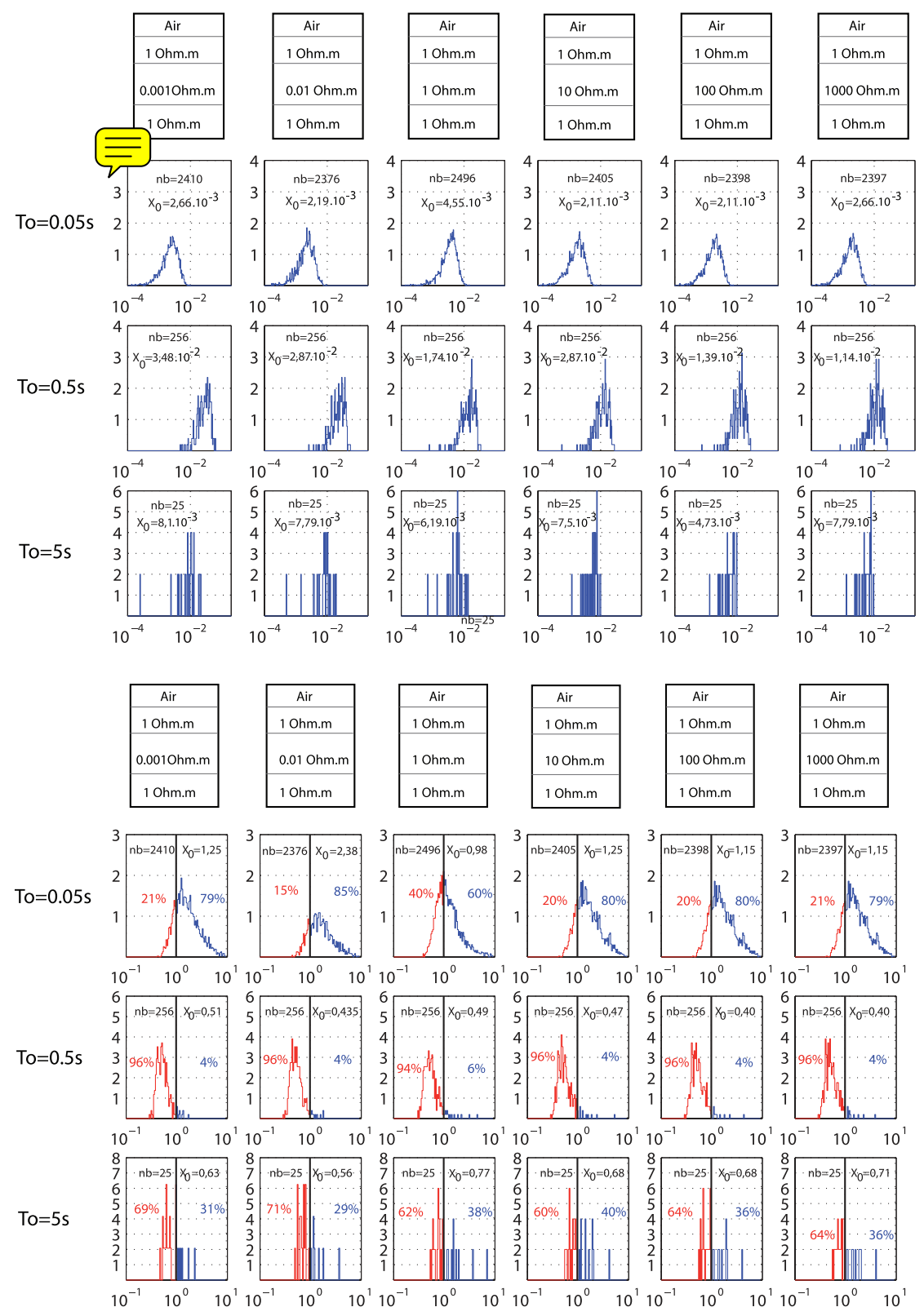

Figure $61 \mathrm{D}$ case: synthetic data. K is 6. Histogram of relative true errors $\left|\Delta Z x y^{o b s}\right| /\left|Z x y^{o b s}\right|$ for 3 periods of estimation $(0.05 s, 0.5 s, 5 s)$, and 5 resistivity models $\left(\rho 2=10^{-2}, 10^{-1}, 1,10^{1}, 10^{2}, 10^{3} \Omega . m\right)$. $X_{0}$ is the most likely value.

Figure 7 1D case: synthetic data. Same as Fig. 5 for BIRRP errors normalized by true errors $\left|\Delta Z x y^{\text {birrp }}\right| /\left|\Delta Z x y^{o b s}\right|$. Red and blue curves as above in Fig. 2.

\section{Time variability}

As in the homogeneous case, let us consider the correlation values between true and estimated errors (see Table 3):

- For the shortest period $T_{0}=0.05 \mathrm{~s}$, both $C_{i j}^{o b s / b i r p}$ values are very low $(-0.08$ to 0.78$)$. The longer periods show higher correlations but never higher than 0.83 . As for the homogeneous case, the variability of the true errors on the MT tensor is unsatisfyingly represented by $\Delta Z^{\text {birrp }}$.

- For the shortest period $T_{0}=0.05 \mathrm{~s}$, most $C_{i j}^{\text {obs/est }}$ values are low $(-0.09$ to 0.74$)$. For periods $T_{0}=0.5 \mathrm{~s}$ and $T_{0}=$
$5 \mathrm{~s}$, correlations values are high $(\geq 0.81)$. At these periods, one can say that error tensor estimates $\Delta Z^{\text {est }}$ behave similarly to the true errors $\Delta Z^{o b s}$. Besides, we note a significant difference between the components: the $x y$ values range from $0.95-0.99$, while the $y x$ values are systematically lower (half of them between $0.81-0.89$ ).

\section{Behaviour of error estimates with Gaussian noise}

In order to test the behaviour of error estimates with electric noise, different levels of noise is injected on synthetic electric 
Figure 8 1D case: synthetic data. Same as Fig. 6 for our proposed error tensor normalized by true errors $\left|\Delta Z x y^{e s t}\right| /\left|\Delta Z x y^{o b s}\right|$.

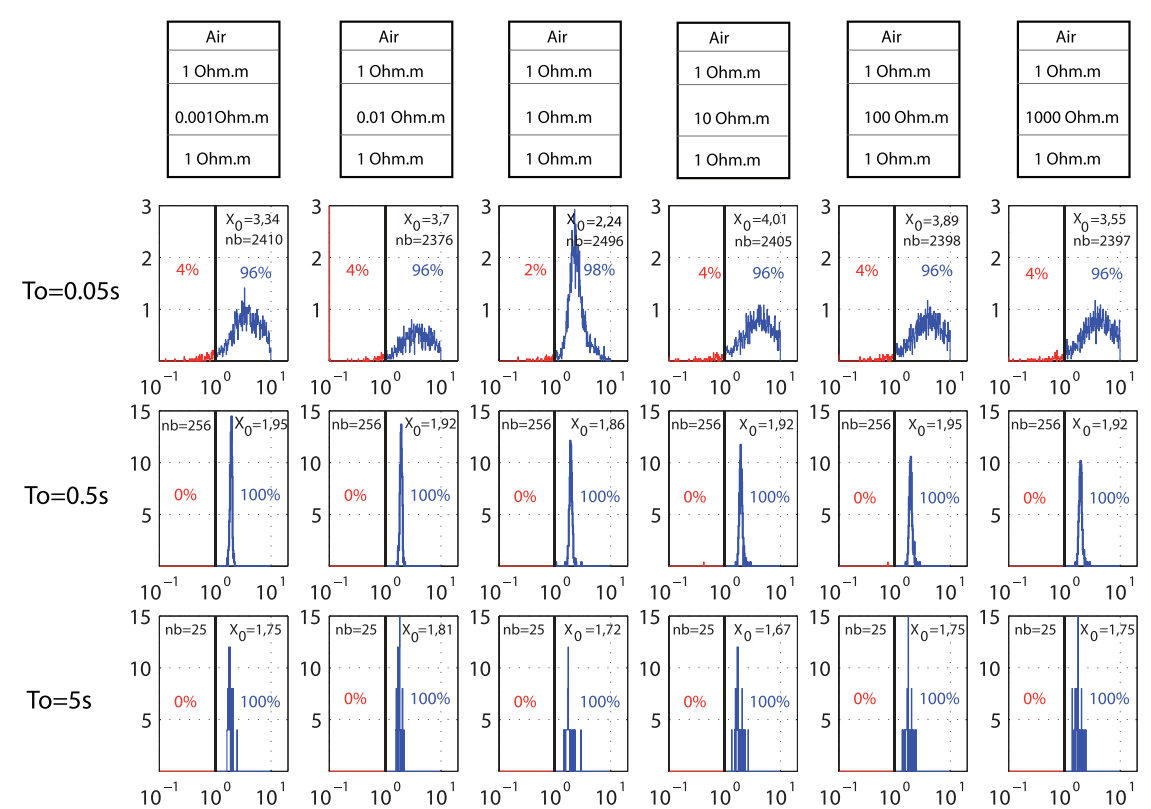

Table 3 1D CASE: as for Table 2 but for synthetic data with $K$ fixed to 6

\begin{tabular}{llllll}
\hline$T_{0}[\mathrm{~s}]$ & $\rho_{2}[\Omega . \mathrm{m}]$ & $C_{x y}^{\text {obs/est }}$ & $C_{y x}^{\text {obs/est }}$ & $C_{x y}^{\text {obs/birrp }}$ & $C_{y x}^{\text {obs/birrp }}$ \\
\hline \multirow{2}{*}{0.05} & $10^{-2}$ & 0.15 & 0.05 & 0.06 & 0.13 \\
& $10^{-1}$ & 0.74 & 0.67 & 0.78 & 0.78 \\
& $10^{-1}$ & 0.20 & 0.17 & 0.27 & 0.48 \\
& $10^{0}$ & -0.08 & 0.04 & -0.05 & -0.08 \\
& $10^{1}$ & -0.09 & 0.05 & -0.05 & -0.08 \\
& $10^{2}$ & -0.09 & 0.05 & -0.05 & -0.08 \\
& $10^{-2}$ & 0.99 & 0.88 & 0.83 & 0.63 \\
& $10^{-1}$ & 0.99 & 0.88 & 0.84 & 0.63 \\
& $10^{0}$ & 0.99 & 0.89 & 0.84 & 0.62 \\
& $10^{1}$ & 0.99 & 0.92 & 0.83 & 0.60 \\
& $10^{2}$ & 0.99 & 0.92 & 0.83 & 0.58 \\
& $10^{3}$ & 0.99 & 0.92 & 0.83 & 0.58 \\
& $10^{-2}$ & 0.99 & 0.97 & 0.75 & 0.72 \\
& $10^{-1}$ & 0.99 & 0.98 & 0.72 & 0.65 \\
& $10^{0}$ & 0.97 & 0.94 & 0.57 & 0.54 \\
& $10^{1}$ & 0.95 & 0.83 & 0.31 & 0.45 \\
$10^{2}$ & 0.95 & 0.81 & 0.25 & 0.46 \\
$10^{3}$ & 0.95 & 0.81 & 0.25 & 0.45 \\
\hline
\end{tabular}

field $e_{i}^{s}(t)$ time series such as

$e_{i}^{N}(t)=e_{i}^{s}(t)+N\left(t, \sigma_{N}\right)$, iso;

where $\sigma_{N}$ is the standard deviation of the noise, which we set proportional to the electric field standard deviation $\sigma_{e_{1}^{s}}$ such as $\sigma_{N}=a \sigma_{e_{i}^{s}}$ and $a$ is defined as the noise level. Comparison between the 3 types of errors are made as for the noise-free data case. As the discussion on the statistical nature of EM noise is still open, we choose to inject simple Gaussian noise on the electric field time series.

Different noise levels were injected on the electric field, respectively corresponding to values of $a$ (equal to $0.2,0.5,0.1$, $0.15,0.2,0.25,0.3$ or 0.5 ). First, the statistical behaviour of the estimates is briefly synthetized by standard deviations and median values of observed and estimated quantities. Values associated with relative true error populations $\left|\Delta Z_{i j}^{\text {obs }}\right| /\left|Z_{i j}^{\text {obs }}\right|$ increase with the noise level (see Fig. 9). For $a=0$, one can find the results observed in the noise-free case. At $T_{0}=0.05 \mathrm{~s}$, relative observed errors increase strongly with the noise level. Again, error tensor estimates are overestimated by a reasonable factor while BIRRP error estimates can be strongly underestimated (not represented here).

Second, $C_{i j}^{\text {obs } / \text { birrp }}$ values are very low at all periods $\left(T_{0}=\right.$ $0.05 \mathrm{~s}, 0.5 \mathrm{~s}, 5 \mathrm{~s}$ ) for both non-diagonal components and varies with $a . C_{i j}^{\text {obs } / e s t}$ increase as a function $a$ at different rates. For $T_{0}=0.5 \mathrm{~s}, 5 \mathrm{~s}$, values are always superior to 0.85 . At $T_{0}=$ $0.05 \mathrm{~s}, C_{i j}^{o b s / e s t}$ are very low on the $y x$ component for values of $a$ lower than 0.1 and increase strongly with increasing noise $a$. A possible explanation for this correlation is that when a low noise level is added to the electric field, errors are principally caused by bias in the magnetic field, propagated to the electric field with maximum correlation. When higher levels of Gaussian noise are present, the scatter becomes dominated by synthetic noise. While BIRRP's own error estimates fail to correlate with the true errors, the higher the noise level, the more $\Delta Z_{i j}^{\text {est }}$ correlates with $\Delta Z_{i j}^{\text {obs }}$. 


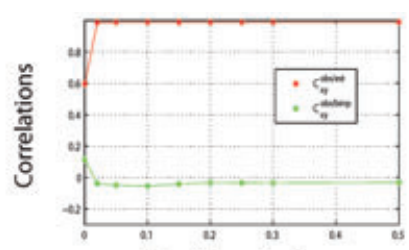

Noise/Signal ratio

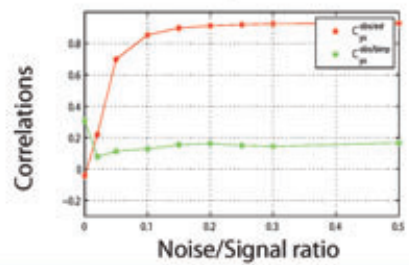

Noise/Signal ratio

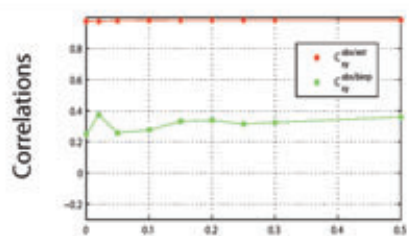

Noise/Signal ratio
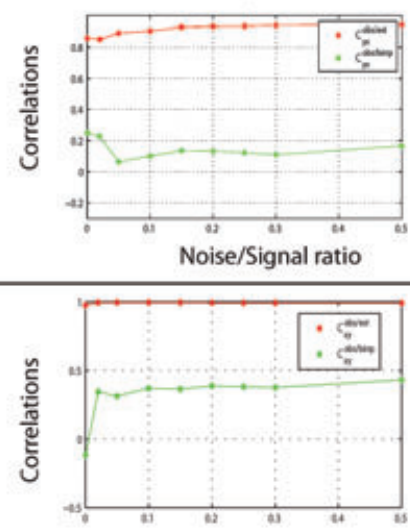

$T=5 s$

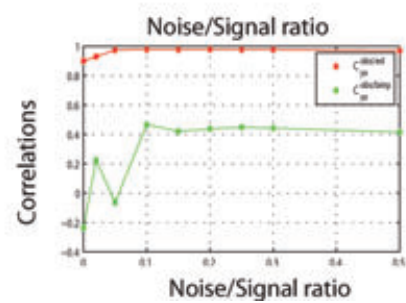

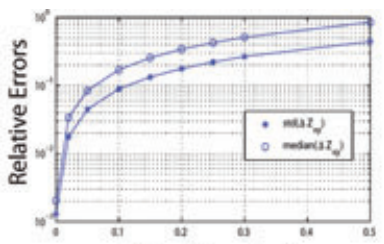

Noise/Signal ratio

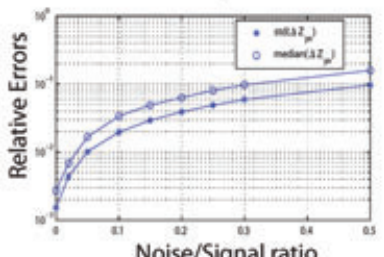

Noise/Signal ratio

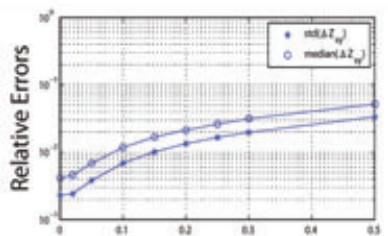

Noise/Signal ratio
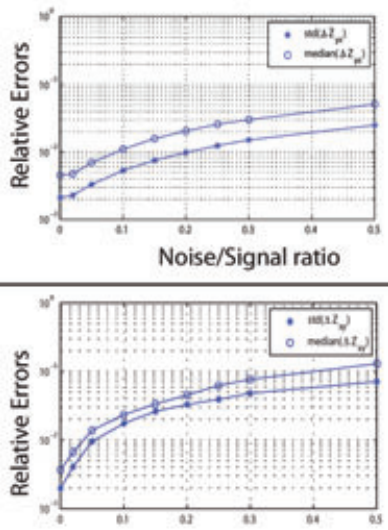

Noise/Signal ratio

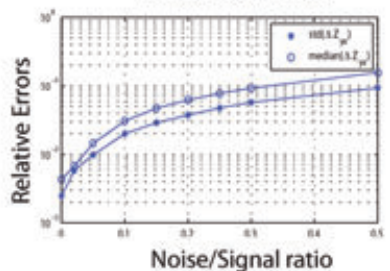

Figure 9 Homogeneous case: synthetic data with a different noise level injected, controlled by parameter $a$ (noise/signal ratio), on the electric field. Left: correlation coefficients between the true and estimated error time-series as a function of $a$ at different periods $T_{0}$ of estimation. $C_{i j}^{\text {obs/est }}$ and $C_{i j}^{o b s / b i r r p}$ explained in the text. Right: median values and standard deviation of the relative observed error population as a function of $a$.

\section{REAL DATA APPLICATION}

Let us now show tests of the error estimation methodology on a real data set acquired at a site in Vendée (Western France) where subsurface resistivity is reasonably assumed to be constant in time. Time series were recorded for a whole day (with a $1024 \mathrm{~Hz}$ sampling frequency), a sufficiently long record for estimates at periods shorter than $5 \mathrm{~s}$. Consecutive values of $Z$ and $\Delta Z$, were computed over the whole $(e, h)$ time-series using
$K=6$ and $N=250$ for the sliding windows in BIRRP. The comparison between the variability and the error estimates is a more useful way to estimate the quality in the real data case, without any a priori knowledge of the subsurface resistivity structure. Besides, the behaviour of $\Delta Z^{\text {est }} / \Delta Z^{\text {obs }}$ cannot be reached on real data since the true error $\Delta Z^{\text {obs }}$ is unknown; this can only be discussed by comparison with the tests on synthetic data. 


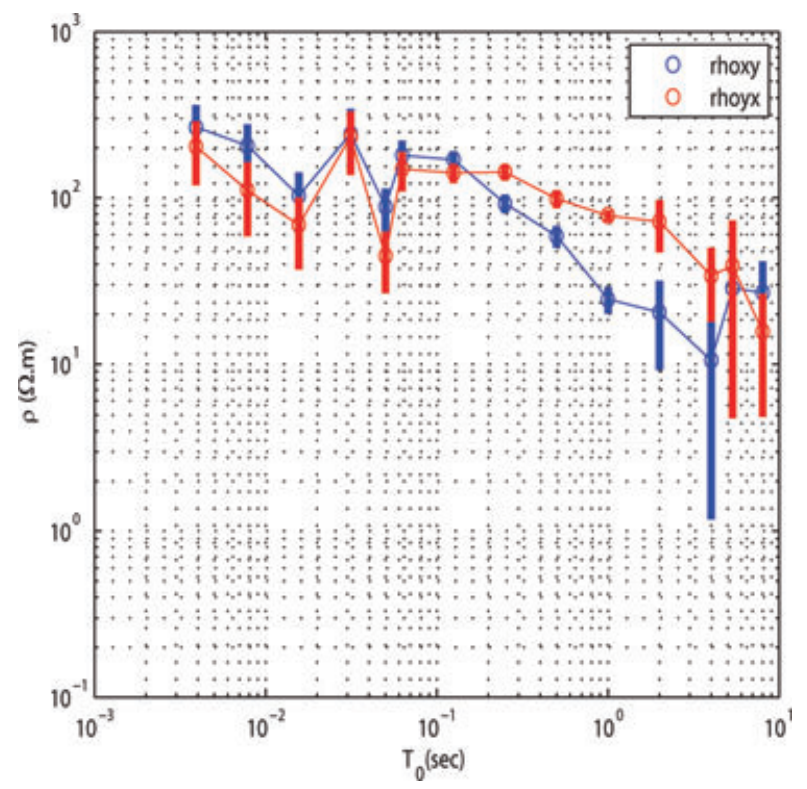

Figure 10 Real data case: noisy MT sounding. Presentation of MT results at the 3 periods of interest and other additional periods: apparent resistivities and associated error bars obtained with the error tensor estimator. Values are obtained with a real observed MT time-series over one day of data. Daily medians of successive values are represented. $\mathrm{K}$ (the number of periods To contained in each delete-one) is fixed to 6 .

\section{Typical magnetotelluric (MT) sounding}

First, we present a typical MT sounding realized with periods going from $3.9 \mathrm{~ms}$ to $8 \mathrm{~s}$ from our real data set, including the 3 periods used in our synthetic tests $\left(T_{0}=0.05 \mathrm{~s}, 0.5 \mathrm{~s}\right.$ and $\left.5 \mathrm{~s}\right)$. These sounding quantities are obtained from the rotation of impedance tensor estimates in geo-electrical directions. Typical apparent resistivities $\rho_{x y}$ et $\rho_{y x}$ are shown with associated error bars computed from the median values of error tensor estimates over the daily estimates.

Resistivities are of a hundred $\Omega . m$ at short periods and both $\rho_{x y}$ and $\rho_{y x}$ are similar. Both decrease at longer periods $\left(T_{0}>0.5 s\right)$ while $x y$ and $y x$ components differ. This sounding has been done on a fractured granitic area with conductor inclusions.

\section{Statistical behaviour of impedance estimates}

We discuss the median daily relative errors computed for $T_{0}=$ $0.05 \mathrm{~s}, 0.5 \mathrm{~s}$ and $5 \mathrm{~s}$ (see Table 4). First, at $T_{0}=5 \mathrm{~s}$, all components of the tensor show large relative errors, equal or superior to $100 \%$. This reflects the great variability shown on tensor component time series at this period. Both periods $T_{0}=$
Table 4 Real data case. Standard deviations of the absolute impedance estimates $\left|Z^{\text {est }}\right|$ time-series $T_{0}$ compared to the median of absolute associated errors $\Delta Z^{e s t}$ for the three periods $T_{0}$ of observation (respectively 5, 0.5 and $0.05 \mathrm{~s}$ ). Both values are normalized to the median values of impedance estimates

\begin{tabular}{llll}
\hline$T_{0}[\mathrm{~s}]$ & 0.05 & 0.5 & 5 \\
\hline median $\left(\left|\Delta Z_{x x}^{\text {sst }}\right| /\left|Z_{x x}^{\text {est }}\right|\right)$ & $18 \%$ & $13 \%$ & $137 \%$ \\
median $\left(\left|\Delta Z_{x y}^{\text {est }}\right| /\left|Z_{x y}^{\text {est }}\right|\right)$ & $34 \%$ & $20 \%$ & $91 \%$ \\
median $\left(\left|\Delta Z_{y x}^{\text {st }}\right| /\left|Z_{y x}^{\text {est }}\right|\right)$ & $41 \%$ & $60 \%$ & $144 \%$ \\
median $\left(\left|\Delta Z_{y y}^{\text {est }}\right| /\left|Z_{y y}^{\text {est }}\right|\right)$ & $25 \%$ & $15 \%$ & $97 \%$ \\
$\operatorname{std}\left(\left|Z_{x x}^{\text {est }}\right|\right) /\left|Z_{x x}^{\text {est }}\right|$ & $10 \%$ & $8.5 \%$ & $30 \%$ \\
$\operatorname{std}\left(\left|Z_{x y}^{\text {est }}\right|\right) /\left|Z_{x y}^{\text {est }}\right|$ & $28 \%$ & $5 \%$ & $50 \%$ \\
$\operatorname{std}\left(\left|Z_{y x}^{\text {est }}\right|\right) /\left|Z_{y x}^{\text {est }}\right|$ & $13 \%$ & $187 \%$ & $280 \%$ \\
$\operatorname{std}\left(\left|Z_{y y}^{\text {est }}\right|\right) /\left|Z_{y y}^{\text {est }}\right|$ & $7 \%$ & $7 \%$ & $34 \%$ \\
\hline
\end{tabular}

$0.05 \mathrm{~s}$ and $T_{0}=0.5 \mathrm{~s}$ have relative errors reaching several tens of percents. $\Delta Z_{y x}$ shows the highest values.

\section{Daily variation}

As proposed by Eisel and Egbert (2001), day-to-day variability would be the best way to estimate impedance estimate precision for a real data set with very long time-series. We apply this variability criterion on a consequently shorter time scale: we study the variability of successive estimates (each one is associated with a duration $\mathrm{D}$ of signal) over a day and compare it with the error estimates based upon residuals. Reliable error estimates must provide the upper and lower bounds of the $Z$ estimate time variations. We know that the subsurface geo-electrical structure does not change during the period of analysis, so that the daily variation is uniquely due to anthropic EM noise, measurement errors and non-stationarity of the source. We define the lower and upper bounds $Z^{-}$and $Z^{+}$of the confidence interval (see the Appendix). We will consider the errors $\Delta Z^{\text {est }}$ to be reliable when their associated confidence interval $\left(\left[Z^{-}: Z^{+}\right]\right)$allows the MT user to take a single value that will remain valid for the whole day.

We will now observe if this condition is respected by comparing the whole-day median and the standard deviation of the $Z$ estimates for all components (as an example, Fig. 11 shows the variations of $Z_{x x}$ bounds over the duration of the dataset). Our results (see Table 4) show that one of the three period's $T_{0}$, strictly meets this condition: for $T_{0}=0.05 \mathrm{~s}$, median values of errors overestimates the standard deviation of $Z$ estimates. Similarly, for $T_{0}=0.5 \mathrm{~s}$ and $T_{0}=5 \mathrm{~s}$, the 

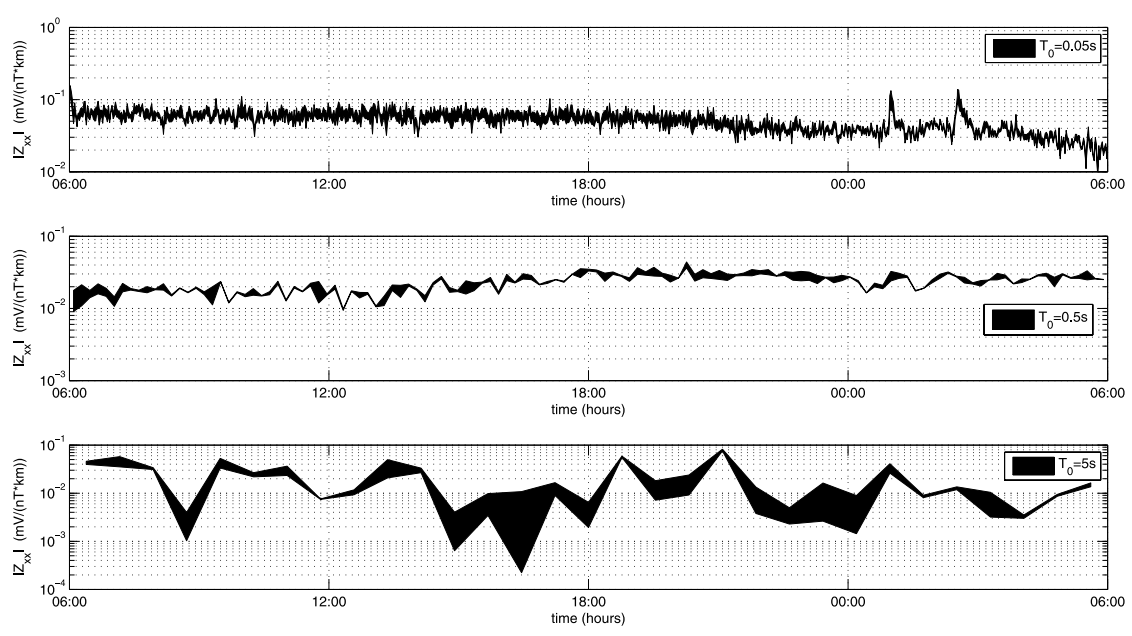

Figure 11 Real data case. Time series of the modulus of the confidence bounds of component $Z_{x x}$ (in black) for 3 periods $T_{0}$ of estimation $(0.05 s, 0.5 s, 5 s)$. condition is met on components $x x, x y$ and $y y$ and confidence intervals can be used as the actual bounds of $Z$ over the day. However, errors underestimates the standard deviations on $y x$ for these periods.

We also observe this condition on apparent resistivities estimates. Tensor estimates $Z$ and associated error tensor $\Delta Z$ are now rotated into the $Z$ principal axis and become $Z^{\prime}$ and $\Delta Z^{\prime}$ (see Appendix for details). From antidiagonal components of $Z^{\prime}$ and $\Delta Z^{\prime}$, the upper and lower bounds are defined (see Appendix for details) and are transformed in resistivity bounds $\rho^{+}$and $\rho^{-}$. Moreover, from $Z^{\prime}$ and $\Delta Z^{\prime}$, a resitivity tensor $\hat{\rho}$ and an error tensor of resistivity $\Delta \rho$ are deduced. Then values of the standard deviation of resistivity estimate time series are compared with the median value of $\Delta \rho$ for $x y$ and $y x$ components.

For $T_{0}=0.05 \mathrm{~s}$, standard deviations of estimated resistivities are slightly equal to the median of resitivity errors, time series of $\rho_{x y}^{+}$and $\rho_{x y}^{-}$and $\rho_{y x}^{+}$and $\rho_{y x}^{-}$values can be used as resistivity bounds at this period. In contrary, for $T_{0}=0.5 \mathrm{~s}$ and $T_{0}=5 \mathrm{~s}$, standard deviations are significantly larger than the median of resitivity errors (see Table 5). Figure 12 shows that at these periods, confidence bounds do not allow to take one single daily resitivity value.

\section{CONCLUSION}

As an alternative to classical error estimates on MT impedances, we propose a new 'robust' MT error tensor estimate $\Delta Z$, computed from transfer functions from magnetic time-series $h(t)$ and the electric field residuals time-series $\Delta e(t)$, resulting from the impedance tensor estimate. They are computed with the same robust processing code as the impedance $Z$ itself, which allows for easy calculations. Tested on two
Table 5 Real data case. Standard deviations of the absolute resistivity estimates $\rho_{i j}^{\text {est }}$ time-series $T_{0}$ compared to the median of the absolute associated errors $\Delta \rho_{i j}^{\text {est }}$ for the three periods $T_{0}$ of observation (respectively $5,0.5$ and $0.05 \mathrm{~s}$ ). All values are normalized to the median values of resistivity estimates

\begin{tabular}{llll}
\hline$T_{0}[\mathrm{~s}]$ & 0.05 & 0.5 & 5 \\
\hline median $\left(\left|\Delta \rho_{x y}^{e s t}\right| /\left|\rho_{x y}^{e s t}\right|\right)$ & $12 \%$ & $11 \%$ & $83 \%$ \\
$\operatorname{median}\left(\left|\Delta \rho_{y x}^{e s t}\right| /\left|\rho_{y x}^{e s t}\right|\right)$ & $14 \%$ & $8 \%$ & $73 \%$ \\
$\operatorname{std}\left(\left|\rho_{x y}^{e s t}\right|\right) /\left|\rho_{x y}^{e s t}\right|$ & $11 \%$ & $81 \%$ & $128 \%$ \\
$\operatorname{std}\left(\left|\rho_{y x}^{\text {est }}\right|\right) /\left|\rho_{y x}^{\text {est }}\right|$ & $13 \%$ & $63 \%$ & $99 \%$ \\
\hline
\end{tabular}

synthetic homogeneous and $1 \mathrm{D}$ synthetic data sets, this procedure yields more reliable estimates on the modulus than the errors based on the Jackknife variance. This is clear on synthetic data sets:

- First, the error tensor 'method' overestimates true errors in reasonable proportions (of factor 2).

- Second, with the exception of the shortest period studied here, all correlations between the true and the new 'robust' errors time-series are quite high (0.9). The variability displayed by error tensor estimates reflects the time variations of true errors, which was not the case with Jackknifebased error estimates. Moreover, we injected several levels of Gaussian noise on the electric field and obtained the following results: new 'robust' error estimates become highly correlated for moderate amounts of noise for all tested periods. This feature is really important for the development of time-lapse MT, where successive estimates are made continuously in time: time variations of error estimates must reflect the true fluctuations in the quality of the tensor. This is a clear improvement compared to previous Jacknife-based 
Figure 12 Real data case. Time series of the confidence interval associated with rotated apparent resistivities $\rho_{x y}$ and $\rho_{y x}$ for 3 periods $T_{0}$ of estimation $(0.05 \mathrm{~s}, 0.5 \mathrm{~s}, 5 \mathrm{~s})$ represented in different grey levels.
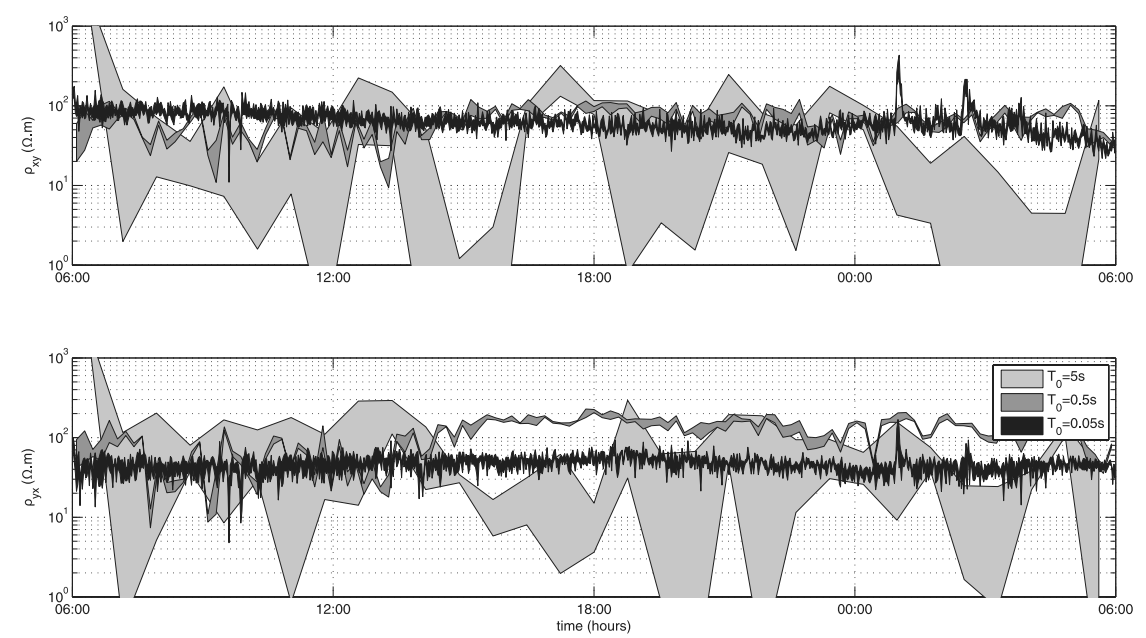

error estimates. This is important in MT for rapid quality control (QC) and to optimize data collection durations.

In a real data case, in order to test the error tensor estimates, one should observe a different condition: considering one time-series of successive impedance estimates, reliable error estimates should englobe their time variations. The 'robust' error tensor values are strictly higher than the daily variation of successive impedance estimates on 1 of our 3 periods of study. In order to compare our results with classical MT apparent resitivities, impedance errors were converted into apparent resistivity errors. The resulting resitivity confidence intervals does not englobe resistivity time variations.

The necessary condition for the development of imaging (in space and time) using MT monitoring of the subsurface (such as volcanoes, hydrocarbon reservoirs or geothermal fields) is the reliability and robustness of both impedances and associated error estimates. The proposed 'robust' errors can be used for such applications, because they provide good correlations with the time variation of the true errors of successive estimates. In addition to the new error estimator, we also propose a methodology to test the transfer function estimator in MT. This procedure can be applied with any MT impedance estimation method. Robustness of the error tensor was shown, at least on its modulus. One valuable addition to this study would be to run the same test with the use of a magnetic remote reference, which can improve results obtained with real data.

Besides, we only used the modulus of the error tensor in this paper but a reliable phase error estimator should be an improvement to the method. As the phase of the impedance tensor is less sensitive to near-surface distortions, it is often necessary to use it for inversion. Phase errors could also be used as a weighting factor in the inversion process. This will be the subject of a future article.

\section{ACKNOWLEDGEMENTS}

We wish to thank the region of Vendée (France) for supporting the survey from which the used real data were extracted. We also thank the survey staff composed of Boualem Bouyahiaoui, Julien Guillemoteau, Maksim Bano and Vincent Allegre.

We benefited from comments by Pascal Tarits, Jacques Zlotnicki, Jean Jacques Schott, Michel Cara, Juanjo Ledo, Aude Chambodut and anonymous reviewers.

Comments and suggestions from two anonymous reviewers have greatly helped to improve this manuscript.

\section{REFERENCES}

Berdichevsky M.N. and Zhdanov M.S. 1984. Advanced Theory of Deep Geomagnetic Sounding. Elsevier, Amsterdam.

Chave A.D. and Jones A.G. 1997. Electric and magnetic field galvanic distortion decomposition of BC87 data. Journal of Geomagnetism and Geoelectricity 49, 4669-4682.

Chave A.D. and Thomson D.J. 1989. Some comments on magnetotelluric response function estimation. Journal of Geophysical Research 94, 14215-14225.

Chave A.D. and Thomson D.J. 2004. Bounded influence estimation of magnetotelluric response functions. Geophysical Journal International 157, 988-1006. 
Chave A.D., Thomson D.J. and Ander M.E. 1987. On the robust estimation of power spectra, coherences, and transfer functions. Journal of Geophysical Research 92, 633-648.

Efron B. 1982. The Jackknife, the Bootstrap and Other Resampling Plans. Society for Industrial and Applied Mathematics, Philadelphia.

Efron B. and Gong. 1983. A leisurely look at the bootstrap the jackknife, and cross-validation. Journal of the American Statistical Association $37,36-48$.

Egbert G.D. 1997. Robust multiple-station magnetotelluric data processing. Geophysical Journal International 130, 475-496.

Egbert G.D. and Booker J. 1986. Robust estimation of geomagnetic transfer functions. Geophysical Journal of the Royal Astronomical Society 87, 173-194.

Eisel M. and Egbert G.D. 2001. On the stability of magnetotelluric transfer function estimates and the reliability of their variances. Geophysical Journal International 144, 65-82.

Gamble T.D., Goubau W.M. and Clarke J. 1979. Magnetotellurics with a remote reference. Geophysics 61, 53-68.

Hanekop O. and Simpson F. 2006. Error propagation in electromagnetic transfer functions: What role for the magnetotelluric method in detecting earthquake precursors? Geophysical Journal International 165, 763-774.

Jones A.G., Chave A.D., Egbert G., Auld D. and Bahr K. 1989. A comparison of techniques for magnetotelluric response function estimation. Journal of Geophysical Research 94, 14201-14213.

Larsen J.C. 1980. Electromagnetic response functions from interrupted and noisy data. Jurnal of Geomagnetics and Geoelectrics 32, Supp. I, SI89-SI103.

Sims W.E., Bostick F.X. and Smith H.W. 1971. The estimation of magnetotelluric impedance tensor elements from measured data. Geophysics 36, 938-942.

Vozoff K. 1987. The Magnetotelluric Method. Electromagnetic methods in applied Geophysics: Volume 2, Application, SEG.

Wannamaker P.E. 1999. Affordable magnetotellurics: Interpretation in natural environments, three-dimensional electromagnetics. Society of Exploration Geophysicists, 349-374.

Ward S.H. and Hohmann G.W. 1987. Electromagnetic Theory for Geophysical Applications. Electromagnetic methods in applied Geophysics: Volume 1, Theory, SEG.

Zhang Y. and Paulson K.V. 1997. Enhancement of signal-to-noise ratio in natural-source transient MT data with wavelet transform. Pure and Applied Geophysics 149, 405-419.

\section{APPENDIX}

As only the module of the error tensor is defined, one should consider all phase values in order to define confidence intervals on rotated impedances. First, we define a family $Z_{i j}^{*}$ of $Z_{i j}$ components, covering the full range of possible phases, such as:

$Z_{i j}^{*}=\left\{Z_{i j}+\left|\Delta Z_{i j}\right| \mathrm{e}^{i \alpha}\right\}$,

where $\mathrm{i}=\sqrt{-1}$ and where $\alpha$ covers 0 to $2 \pi$. Thus, the bounds of the confidence interval for each component $Z_{i j}$ (respectively $Z_{i j}^{-}$and $Z_{i j}^{+}$) are defined by minimum and maximum modulus values in the family of $Z_{i j}^{*}$. In addition, with these bounds, we define two matrices of dimension $2 \times 2$ of confidence intervals $Z^{-}$and $Z^{+}$.

We now consider the case where the tensor is rotated through its principal axes. Let $\theta$ be the strike angle determined on the basis of the impedance estimate $Z$, from which we define a rotation matrix:

$R_{\theta}=\left[\begin{array}{cc}R_{x x}^{\theta} & R_{x y}^{\theta} \\ R_{y x}^{\theta} & R_{y y}^{\theta}\end{array}\right]$.

Then, the tensor is rotated with $R_{\theta}$ and each rotated component is associated with a family of values :

$Z_{x x, \theta}=R_{x x}^{\theta} Z_{x x}^{*}+R_{x y}^{\theta} Z_{y x}^{*}$

$Z_{x y, \theta}=R_{x x}^{\theta} Z_{x y}^{*}+R_{x y}^{\theta} Z_{y y}^{*}$

$Z_{y x, \theta}=R_{y x}^{\theta} Z_{x x}^{*}+R_{y y}^{\theta} Z_{y x}^{*}$

$Z_{y y, \theta}=R_{y x}^{\theta} Z_{x y}^{*}+R_{y y}^{\theta} Z_{y y}^{*}$.

From the maximum and minimum values of the modulus of each family component, the tensors of upper and lower bounds of confidence interval $Z_{\theta}^{+}$and $Z_{\theta}^{-}$are defined. Thus, they are transformed in the resistivity bound matrix from which values $\rho_{i j}^{+}$and $\rho_{i j}^{-}$are taken. 